\title{
Reformulation of Frequency Based Substructuring Method Considering Elastic Joints According to Sherman-Morrison-Woodbury Formula
}

\author{
Jiangpan CHEN, Yi LIU, Weiwen ZHANG, Yan LIU*, Dong WANG, Limin SUN
}

\begin{abstract}
The frequency based substructuring method considering elastic joints (FBSM-CEJ) is reformulated according to Sherman-Morrison-Woodbury Formula (SMWF) in this paper, the derivation process of which is more concise and the order of the matrix that requires inversion in the corresponding derivation result is lower comparing to the existing FBSM-CEJ. Meanwhile, the reformulated FBSM-CEJ possesses more applicability and operability that can be used to directly and efficiently calculate the frequency response function (FRF) matrix of complex structure no matter the impedance matrix of the elastic joints is singular or not. Last but not least, via using none-mass spatial beam element to simulate the dynamic properties of elastic joints, the performance of the reformulated FBSM-CEJ is verified through numerical simulation. All the achievements obtained from this work will provide a theoretical basis for analysing the dynamic properties of a complex structure considering elastic joints.
\end{abstract}

Keywords: elastic joints; frequency based substructuring method; frequency response function; Sherman-Morrison-Woodbury Formula; spatial beam element

\section{INTRODUCTION}

In pace with the rapid development of science and technology, the engineering structure, such as aircraft and spacecraft, is becoming increasingly complicated. Thus, instead of traditional finite element method (FEM), dynamic substructure method is more and more widely used to analyse the dynamic properties of the complex engineering structure, [1-2]. According to solution domain, the dynamic substructure method can be divided into component mode synthesis (CMS) in time domain and FBSM in frequency domain. With regard to CMS, there are three different widely used methods, namely fixed interface component mode synthesis (FiICMS), free interface component mode synthesis (FrICMS) and hybrid interface component mode synthesis (HyICMS), [3]. In regard to FBSM, both impedance coupling method (ICM) and receptance coupling method (RCM) are frequently used in actual engineering $[4,5]$. Based on the structural dynamics theory, the frequently used dynamic substructure methods can be demonstrated as in Fig. 1.

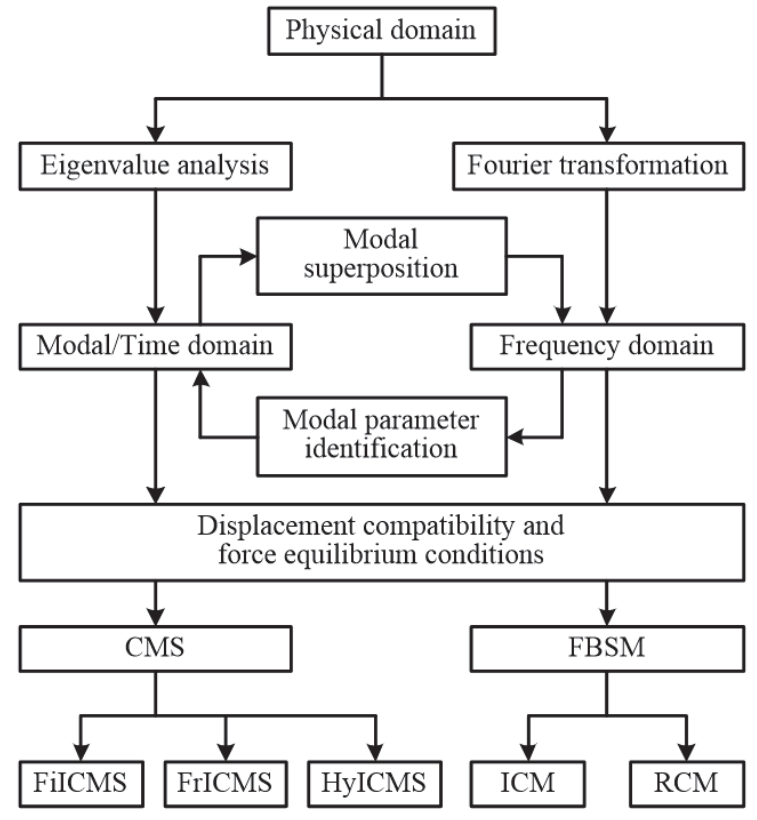

Figure 1 Schematic diagram of dynamic substructure method
Generally, the sophisticated CMS is appropriate for dealing with the substructure synthesis between the theoretical models established via FEM. Along with the continuous improvement of both test equipment and test method, the substructure synthesis between test-test models or test-theoretical models is becoming more and more common, where the test models are obtained by experimental test. The FBSM was more suitable for dealing with the substructure synthesis between test-test models or test-theoretical models, [6]. The ICM is the initially proposed method of FBSM. Due to the inverse operation of the full FRF matrix of the substructure being required, both the calculation accuracy and efficiency of ICM are poor. Additionally, the ICM will be no longer in force when the full FRF matrix of the substructure is singular [7]. Since only the FRF matrix of the interfacial degree of freedoms (DOFs) of the substructure is needed to be inversed, the calculation accuracy and efficiency of the RCM, another method of FBSM and developed by Jetmundsen et al., are dramatically improved comparing with ICM, [8]. However, the RCM is only suitable for dealing with the substructure synthesis between two independent substructures. Based on the RCM, a more widely used method named generalized receptance coupling method (GRCM), was present to deal with the substructure synthesis among several non-independent or independent substructures by Ren et al., [9]. One significant point to be reminded is that the application area of GRCM is only within the situation when all the joints between substructures are rigid. However, most of the joints existing in actual engineering structure are elastic, [10]. Then, according to GRCM, the existing FBSM-CEJ is formulated via considering all the elastic joints between two substructures as an independent substructure [11-13]. Nevertheless, the derivation process of the existing FBSMCEJ involves a large number of matrix operations, and the corresponding derivation result is incapable of synthesizing the substructures connected via elastic joints with singular impedance matrix directly.

Meanwhile, how to reasonably simulate the dynamic properties of elastic joints is an important factor affecting the accuracy of FBSM-CEJ. In references $[3,4,10]$ and [14], 6-DOFs scalar spring system was employed to 
simulate the elastic joints for investigating the FBSM-CEJ. With regard to this simulation method, nevertheless, the stiffness coupling between DOF of the elastic joints node was not taken into consideration, which is quite different from the elastic joints in actual engineering. Zou [15] and Li [16] believed that comparing with 6-DOFs scalar spring system, the stiffness matrix of spatial beam element was more suitable for simulating the stiffness of the elastic joints because of the existing stiffness coupling between the vertical translation and bending DOF of spatial beam element node.

The remainder of this article proceeds as follows: section 2 briefly introduces the existing FBSM-CEJ; section 3 demonstrates the reformulation of FBSM-CEJ according to SMWF, and indexes the advantages of the reformulated FBSM-CEJ; section 4 describes the use of non-mass spatial beam element to simulate the elastic joints; section 5 validates the reformulated FBSM-CEJ through numerical simulation; section 6 summarizes the paper and states the conclusions drawn from this work; finally, section 7 lists the references.

\section{BRIEF INTRODUCTION OF EXISTING FBSM-CEJ}

As mentioned above, the existing FBSM-CEJ is derived based on the GRCM, in which all the substructures are required to be considered as a substructure system for processing, [9]. The schematic diagram, used to derive the existing FBSM-CEJ, can be illustrated as in Fig. 2, [9-10].

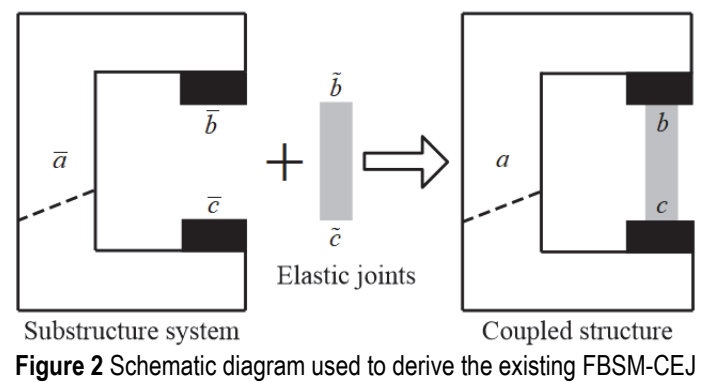

In Fig. 2, $\bar{a}$ and $a$ denote the internal DOFs of the substructure system and coupled structure, respectively, $(\bar{b}, \bar{c}),(\tilde{b}, \tilde{c})$ and $(b, c)$ yield the interfacial DOFs of the substructure system, elastic joints and coupled structure, respectively. As a consequence, the FRF matrix of the substructure system and the impedance matrix of the elastic joints can be defined as follows:

$$
\begin{aligned}
& {\left[\begin{array}{l}
x_{\bar{a}} \\
x_{\bar{b}} \\
\boldsymbol{x}_{\bar{c}}
\end{array}\right]=\left[\begin{array}{lll}
H_{\bar{a} a} & H_{\bar{a} \bar{b}} & H_{\overline{a c}} \\
H_{\bar{b} \bar{a}} & H_{\overline{b b}} & H_{\bar{b} \bar{c}} \\
H_{\overline{c a}} & H_{\bar{c} \bar{b}} & H_{\overline{c c}}
\end{array}\right]\left[\begin{array}{l}
f_{\bar{a}} \\
f_{\bar{b}} \\
f_{\bar{c}}
\end{array}\right]=\bar{H}\left[\begin{array}{l}
f_{\bar{a}} \\
f_{\bar{b}} \\
f_{\bar{c}}
\end{array}\right]} \\
& {\left[\begin{array}{l}
f_{\tilde{b}} \\
f_{\tilde{c}}
\end{array}\right]=\left[\begin{array}{ll}
Z_{\tilde{b} \tilde{b}} & Z_{\tilde{b} \tilde{c}} \\
Z_{\tilde{c} \tilde{b}} & Z_{\tilde{c} \tilde{c}}
\end{array}\right]\left[\begin{array}{l}
x_{\tilde{b}} \\
x_{\tilde{c}}
\end{array}\right]=\tilde{Z}\left[\begin{array}{l}
x_{\tilde{b}} \\
x_{\tilde{c}}
\end{array}\right]}
\end{aligned}
$$

where $\boldsymbol{x}, \boldsymbol{f}, \boldsymbol{H}$ and $\boldsymbol{Z}$ represent the displacement vector, force vector, FRF matrix and impedance matrix, respectively, all the subscripts are corresponding to them demonstrated in Fig. 2. Meanwhile, $\overline{\boldsymbol{H}}$ and $\tilde{\boldsymbol{Z}}$ yield the
FRF matrix and impedance matrix of substructure system and elastic joints respectively.

Via introducing the conditions of force equilibrium and displacement compatibility, the FRF matrix of the coupled structure $\boldsymbol{H}_{\boldsymbol{E}}$ can be illustrated as, $[12,13]$ :

$$
\boldsymbol{H}_{E}=\overline{\boldsymbol{H}}-\left[\begin{array}{cc}
\boldsymbol{H}_{\bar{a} \bar{b}} & \boldsymbol{H}_{\overline{a c}} \\
\boldsymbol{H}_{\overline{b b}} & \boldsymbol{H}_{\bar{b} \bar{c}} \\
\boldsymbol{H}_{\bar{c} \bar{b}} & \boldsymbol{H}_{\bar{c} \bar{c}}
\end{array}\right] \Delta_{E}{ }^{-1}\left[\begin{array}{cc}
\boldsymbol{H}_{\bar{a} \bar{b}} & \boldsymbol{H}_{\overline{a c}} \\
\boldsymbol{H}_{\overline{b b}} & \boldsymbol{H}_{\bar{b} \bar{c}} \\
\boldsymbol{H}_{\bar{c} \bar{b}} & \boldsymbol{H}_{\bar{c} \bar{c}}
\end{array}\right]^{\mathrm{T}}
$$

where the expression of the matrix $\Delta_{E}$ is given by:

$$
\Delta_{E}=\left[\begin{array}{ll}
Z_{\tilde{b} \tilde{b}} & Z_{\tilde{b} \tilde{c}} \\
Z_{\tilde{c} \tilde{b}} & Z_{\tilde{c} \tilde{c}}
\end{array}\right]^{-1}+\left[\begin{array}{ll}
H_{\overline{b b}} & H_{\bar{b} \bar{c}} \\
H_{\bar{c} \bar{b}} & H_{\overline{c c}}
\end{array}\right]
$$

By observing Eq. (3) and Eq. (4), a significant point that should be reminded is that the existing FBSM-CEJ will be no longer applicable when the impedance matrix of the elastic joints is singular.

\section{REFORMULATION OF THE FBSM-CEJ}

The schematic diagram illustrated in Fig. 2 will be used to reformulate FBSM-CEJ since the reformulation process is based on the GRCM as well. According to Eq. (1) and Eq. (2), the FRF matrix of the substructure system $\overline{\boldsymbol{H}}$ and the impedance matrix of the elastic joints $\tilde{\boldsymbol{Z}}$ can be obtained as follows:

$$
\begin{aligned}
& \bar{H}=\left[\begin{array}{lll}
H_{\bar{a} a} & H_{\bar{a} \bar{b}} & H_{\overline{a c}} \\
H_{\bar{b} \bar{a}} & H_{\overline{b b}} & H_{\bar{b} \bar{c}} \\
H_{\overline{c a}} & H_{\bar{c} \bar{b}} & H_{\overline{c c}}
\end{array}\right] \\
& \tilde{Z}=\left[\begin{array}{ll}
Z_{\tilde{b} \tilde{b}} & Z_{\tilde{b} \tilde{c}} \\
Z_{\tilde{c} \tilde{b}} & Z_{\tilde{c} \tilde{c}}
\end{array}\right]
\end{aligned}
$$

Matrix extension will be applied on $\tilde{\boldsymbol{Z}}$ firstly so that:

$\boldsymbol{Z}=\boldsymbol{P} \tilde{Z} \boldsymbol{Q}=\left[\begin{array}{ccc}0 & 0 & 0 \\ 0 & Z_{\tilde{b} \tilde{b}} & Z_{\tilde{b} \tilde{c}} \\ 0 & Z_{\tilde{c} \tilde{b}} & Z_{\tilde{c} \tilde{c}}\end{array}\right]$

where $\boldsymbol{P}$ and $\boldsymbol{Q}$ are both extension matrices, the expressions of which are given by:

$$
\begin{aligned}
& \boldsymbol{P}=\left[\begin{array}{ll}
\boldsymbol{0} & \mathbf{0} \\
\boldsymbol{I} & \mathbf{0} \\
\mathbf{0} & \boldsymbol{I}
\end{array}\right] \\
& \boldsymbol{Q}=\left[\begin{array}{lll}
\mathbf{0} & \boldsymbol{I} & \mathbf{0} \\
\mathbf{0} & \mathbf{0} & \boldsymbol{I}
\end{array}\right]
\end{aligned}
$$

where $\boldsymbol{I}$ indexes the identity matrix. By substituting Eq. (7) into Eq. (2), an achievement can be reached that: 


$$
\left[\begin{array}{c}
0 \\
f_{\tilde{b}} \\
f_{\tilde{c}}
\end{array}\right]=\left[\begin{array}{ccc}
0 & 0 & 0 \\
0 & Z_{\tilde{b} \tilde{b}} & Z_{\tilde{b} \tilde{c}} \\
0 & Z_{\tilde{c} \tilde{b}} & Z_{\tilde{c} \tilde{c}}
\end{array}\right]\left[\begin{array}{c}
x_{\tilde{a}} \\
x_{\tilde{b}} \\
x_{\tilde{c}}
\end{array}\right]=Z\left[\begin{array}{c}
x_{\tilde{a}} \\
x_{\tilde{b}} \\
x_{\tilde{c}}
\end{array}\right]
$$

Since the internal DOFs of the substructure have no change after the substructure synthesis, it can be confirmedly obtained that:

$$
x_{\bar{a}} \equiv x_{a}, f_{\bar{a}} \equiv f_{a}
$$

In addition, the conditions of force equilibrium and displacement compatibility are given by:

$$
\begin{aligned}
& {\left[\begin{array}{l}
f_{b} \\
f_{c}
\end{array}\right]=\left[\begin{array}{l}
f_{\bar{b}} \\
f_{\bar{c}}
\end{array}\right]+\left[\begin{array}{l}
f_{\tilde{b}} \\
f_{\tilde{c}}
\end{array}\right]} \\
& {\left[\begin{array}{l}
x_{b} \\
x_{c}
\end{array}\right]=\left[\begin{array}{l}
x_{\bar{b}} \\
x_{\bar{c}}
\end{array}\right]=\left[\begin{array}{l}
x_{\tilde{b}} \\
x_{\tilde{c}}
\end{array}\right]}
\end{aligned}
$$

Thus via substituting Eq. (11) to Eq. (13) into Eq. (1) it can be obtained that:

$$
\left[\begin{array}{l}
f_{a} \\
f_{b} \\
f_{c}
\end{array}\right]=\left[\begin{array}{c}
f_{\bar{a}} \\
f_{\bar{b}} \\
f_{\bar{c}}
\end{array}\right]+\left[\begin{array}{c}
0 \\
f_{\tilde{b}} \\
f_{\tilde{c}}
\end{array}\right]=\bar{H}^{-1}\left[\begin{array}{c}
x_{a} \\
x_{b} \\
x_{c}
\end{array}\right]+\left[\begin{array}{c}
0 \\
f_{\tilde{b}} \\
f_{\tilde{c}}
\end{array}\right]
$$

Then by substituting Eq. (10) into Eq. (14), the relation can be noticed that:

$$
\left[\begin{array}{l}
f_{a} \\
f_{b} \\
f_{c}
\end{array}\right]=\left(\bar{H}^{-1}+Z\right)\left[\begin{array}{l}
x_{a} \\
x_{b} \\
x_{c}
\end{array}\right]
$$

Consequently, the FRF matrix of the coupled structure will be obtained:

$$
\boldsymbol{H}_{\boldsymbol{R}}=\left(\overline{\boldsymbol{H}}^{-1}+\boldsymbol{Z}\right)^{-1}=\left(\overline{\boldsymbol{H}}^{-1}+\boldsymbol{P} \tilde{\boldsymbol{Z}} \boldsymbol{Q}\right)^{-1}
$$

where $\boldsymbol{H}_{\boldsymbol{R}}$ denotes the FRF matrix of the coupled structure.

Observing Eq. (16), the expression of the right side is similar to that of SMWF, which is given by $[17,18]$ :

$$
(\boldsymbol{A}+\boldsymbol{M B N})^{-1}=\boldsymbol{A}^{-1}-\boldsymbol{A}^{-1} \boldsymbol{M}\left(\boldsymbol{B}^{-1}+\boldsymbol{N} \boldsymbol{A}^{-1} \boldsymbol{M}\right)^{-1} \boldsymbol{N} \boldsymbol{A}^{-1}
$$

In Eq. (17), $\boldsymbol{B}$ is a non-singular matrix whereas matrices $\boldsymbol{M}$ and $\boldsymbol{N}$ a re of rectangular shape. Then, observing Eq. (8) and Eq. (9), both $\boldsymbol{P}$ and $\boldsymbol{Q}$ are matrices of rectangular shape. However, the singularity of $\tilde{\boldsymbol{Z}}$ depends on the structural properties of the elastic joints. In order to meet the requirements of the SMWF, singular value decomposition (SVD) will be used to deal with the impedance matrix of the elastic joints. The SVD of $\tilde{\boldsymbol{Z}}$ is given by [19]:

$$
\tilde{\boldsymbol{Z}}=\boldsymbol{U} \boldsymbol{\Delta} \boldsymbol{V}^{\mathrm{T}}
$$

where $\boldsymbol{\Delta}$ is the positive singular value matrix of $\tilde{\boldsymbol{Z}}$, both $\boldsymbol{U}$ and $\boldsymbol{V}$ are orthogonal matrices. Substituting Eq. (18) into Eq. (16), $\boldsymbol{H}_{\boldsymbol{R}}$ can be redefined as:

$$
\boldsymbol{H}_{\boldsymbol{R}}=\left[\overline{\boldsymbol{H}}^{-1}+(\boldsymbol{P} \boldsymbol{U}) \boldsymbol{\Delta}\left(\boldsymbol{V}^{\mathrm{T}} \boldsymbol{Q}\right)\right]^{-1}
$$

Since $\boldsymbol{\Delta}$ is a diagonal matrix and all the elements on the diagonal are positive, $\boldsymbol{A}$ is a non-singular square matrix. Meanwhile, both $\boldsymbol{P} \boldsymbol{U}$ and $\boldsymbol{V}^{\mathrm{T}} \boldsymbol{Q}$ are rectangular matrices. As a consequence, according to Eq. (17), the FRF matrix of the coupled structure can be rewritten as:

$$
\boldsymbol{H}_{\boldsymbol{R}}=\overline{\boldsymbol{H}}-\overline{\boldsymbol{H}} \boldsymbol{P U}\left(\boldsymbol{\Delta}^{-1}+\boldsymbol{V}^{\mathrm{T}} \boldsymbol{Q} \overline{\boldsymbol{H}} \boldsymbol{P U}\right)^{-1} \boldsymbol{V}^{\mathrm{T}} \boldsymbol{Q} \overline{\boldsymbol{H}}
$$

Substituting Eq. (1), Eq. (8) and Eq. (9) into Eq. (20) it can be realized that:

$$
\boldsymbol{H}_{\boldsymbol{R}}=\overline{\boldsymbol{H}}-\left[\begin{array}{ll}
\boldsymbol{H}_{\bar{a} \bar{b}} & \boldsymbol{H}_{\overline{a c}} \\
\boldsymbol{H}_{\bar{b} \bar{b}} & \boldsymbol{H}_{\bar{b} \bar{c}} \\
\boldsymbol{H}_{\bar{c} \bar{b}} & \boldsymbol{H}_{\bar{c} \bar{c}}
\end{array}\right] \boldsymbol{U} \boldsymbol{\Delta}_{\boldsymbol{R}}{ }^{-1} \boldsymbol{V}^{\mathrm{T}}\left[\begin{array}{cc}
\boldsymbol{H}_{\bar{a} \bar{b}} & \boldsymbol{H}_{\overline{a c}} \\
\boldsymbol{H}_{\bar{b} \bar{b}} & \boldsymbol{H}_{\bar{b} \bar{c}} \\
\boldsymbol{H}_{\bar{c} \bar{b}} & \boldsymbol{H}_{\bar{c} \bar{c}}^{\mathrm{T}}
\end{array}\right]^{\mathrm{T}}
$$

where the expression of $\boldsymbol{\Delta}_{\boldsymbol{R}}$ is defined as:

$$
\boldsymbol{\Delta}_{\boldsymbol{R}}=\boldsymbol{\Delta}^{-1}+\boldsymbol{V}^{\mathrm{T}}\left[\begin{array}{ll}
\boldsymbol{H}_{\overline{b b}} & \boldsymbol{H}_{\bar{b} \bar{c}} \\
\boldsymbol{H}_{\bar{c} \bar{b}} & \boldsymbol{H}_{\overline{c c}}
\end{array}\right] \boldsymbol{U}
$$

By observing Eq. (21) and Eq. (3) it can be obtained that the expression of $\boldsymbol{H}_{\boldsymbol{R}}$ is similar to that of $\boldsymbol{H}_{\boldsymbol{E}}$. However, comparing to the existing FBSM-CEJ, the derivation process of $\boldsymbol{H}_{\boldsymbol{R}}$ is more concise and the corresponding derivation result can be used to directly calculate the FRF matrix of a complex structure considering elastic joints with no matter singular or nonsingular impedance matrix. In addition, since $\boldsymbol{\Delta}$ is the positive singular value matrix of $\tilde{\boldsymbol{Z}}$, it can be confirmedly obtained that:

$$
R(\boldsymbol{\Delta}) \leq R(\tilde{\boldsymbol{Z}})
$$

where $R$ yields the order of the matrix and the equality is true only if $\tilde{\boldsymbol{Z}}$ is a non-singular matrix. Via observing Eq. (4), thus, it can be concluded that the order of the matrix that requires inversion in calculating $\boldsymbol{H}_{\boldsymbol{R}}$ is not greater than it in calculating $\boldsymbol{H}_{\boldsymbol{E}}$, namely:

$R\left(\boldsymbol{\Delta}_{\boldsymbol{R}}\right) \leq R\left(\boldsymbol{\Delta}_{\boldsymbol{E}}\right)$

From what have been discussed above it can be concluded that comparing to the existing FBSM-CEJ illustrated in Eq. (3), the reformulated FBSM-CEJ possesses not only higher efficiency, but also more applicability and operability, which can be used to 
directly and higher-efficiently calculate the FRF matrix of a complex structure considering elastic joints with no matter singular or non-singular impedance matrix.

\section{SIMULATION METHOD OF ELASTIC JOINTS}

Since the mass of the elastic joints is far less than that of the structure, the effect of the elastic joints mass on the dynamic properties of the structure is so small that it can be ignored,[15, 16]. As a consequence, the mass matrix of the elastic joints $\tilde{\boldsymbol{M}}$ can be defined as:

$$
\tilde{\boldsymbol{M}}=\mathbf{0}
$$

In references $[15,16]$ it is believed that the stiffness matrix of spatial beam element is more appropriate to simulate the stiffness of the elastic joints instead of 6-DOFs scalar spring system. The stiffness matrix of spatial beam element is given by [20]:

$$
\boldsymbol{K}_{\boldsymbol{b}}=\left[\begin{array}{ll}
\boldsymbol{K}_{b \boldsymbol{b}} & \boldsymbol{K}_{\boldsymbol{b c}} \\
\boldsymbol{K}_{\boldsymbol{c} \boldsymbol{b}} & \boldsymbol{K}_{c c}
\end{array}\right]=\left[\begin{array}{cc}
\boldsymbol{K}_{\boldsymbol{b b}} & \left(\boldsymbol{K}_{c \boldsymbol{b}}\right)^{\mathrm{T}} \\
\boldsymbol{K}_{c b} & \boldsymbol{K}_{c c}
\end{array}\right]
$$

where $\boldsymbol{K}_{\boldsymbol{b}}$ is the stiffness matrix of spatial beam element that is a singular matrix. Meanwhile, the expressions of matrices $\boldsymbol{K}_{\boldsymbol{b} \boldsymbol{b}}, \boldsymbol{K}_{\boldsymbol{c} \boldsymbol{b}}$ and $\boldsymbol{K}_{c c}$ can be found in reference [21]. From the specific expression of $\boldsymbol{K}_{\boldsymbol{b}}$ it is obviously observed that the existence of the stiffness coupling between the vertical translation and bending DOF makes the stiffness matrix of spatial beam element more appropriate to simulate the stiffness of the elastic joints in actual engineering.

Supposing that all the elastic joints are independent of each other, the stiffness matrix of the elastic joints $\tilde{\boldsymbol{K}}$ can be obtained as:

$$
\tilde{\boldsymbol{K}}=\boldsymbol{G}^{\mathrm{T}}\left[\operatorname{diag}\left(\boldsymbol{K}_{\boldsymbol{b}}, \ldots, \boldsymbol{K}_{\boldsymbol{b}}\right)\right] \boldsymbol{G}
$$

where $\boldsymbol{G}$ is the transformation matrix whose expression is defined as:

$$
\boldsymbol{G}=\left[\begin{array}{cccccccc}
\boldsymbol{I}_{6} & \mathbf{0} & \cdots & \mathbf{0} & \mathbf{0} & \mathbf{0} & \cdots & \mathbf{0} \\
\mathbf{0} & \mathbf{0} & \cdots & \mathbf{0} & \boldsymbol{I}_{6} & \mathbf{0} & \cdots & \mathbf{0} \\
\mathbf{0} & \boldsymbol{I}_{6} & \cdots & \mathbf{0} & \mathbf{0} & \mathbf{0} & \cdots & \mathbf{0} \\
\mathbf{0} & \mathbf{0} & \cdots & \mathbf{0} & \mathbf{0} & \boldsymbol{I}_{6} & \cdots & \mathbf{0} \\
\vdots & \vdots & \ddots & \vdots & \vdots & \vdots & \ddots & \vdots \\
\vdots & \vdots & \ddots & \vdots & \vdots & \vdots & \ddots & \vdots \\
\mathbf{0} & \mathbf{0} & \cdots & \boldsymbol{I}_{6} & \mathbf{0} & \mathbf{0} & \cdots & \mathbf{0} \\
\mathbf{0} & \mathbf{0} & \cdots & \mathbf{0} & \mathbf{0} & \mathbf{0} & \cdots & \boldsymbol{I}_{6}
\end{array}\right]
$$

where $\boldsymbol{I}_{6}$ yields the 6-order identity matrix. Meanwhile, assuming the damping of the elastic joints $\tilde{\boldsymbol{C}}$ is Rayleigh damping, namely:

$$
\tilde{\boldsymbol{C}}=\alpha \tilde{\boldsymbol{M}}+\beta \tilde{\boldsymbol{K}}
$$

where $\alpha$ and $\beta$ are the proportional coefficients of Rayleigh damping.

As a consequence, on the basis of using none-mass spatial beam element to simulate the elastic joints, the impedance matrix of the elastic joints can be defined as:

$$
\tilde{\boldsymbol{Z}}=-\omega^{2} \tilde{\boldsymbol{M}}+j \omega \tilde{\boldsymbol{C}}+\tilde{\boldsymbol{K}}=(1+j \omega \beta) \tilde{\boldsymbol{K}}
$$

where $\omega$ and $j$ present the angular frequency and the imaginary unit, respectively.

\section{NUMERICAL SIMULATION}

In this part, based on using the none-mass spatial beam element to simulate the elastic joints, an example structure with elastic joints is designed to verify the performance of the reformulated FBSM-CEJ through comparing the FRF calculation results obtained via the reformulated FBSMCEJ with those achieved by FEM. The schematic diagram of the example structure is demonstrated in Fig. 3.

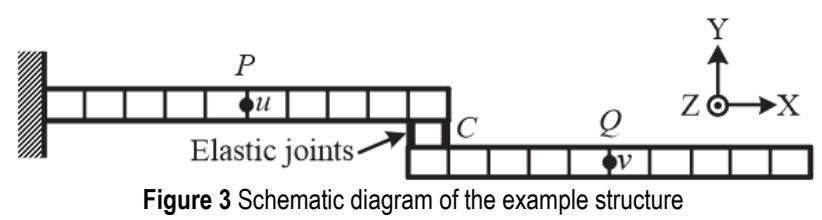

As demonstrated in Fig. 3, the example structure is composed of three parts, namely $P, Q$ and elastic joints $C$. $P$ and $Q$ are exactly the same two rectangular cross-section beams $\left(0.02 \times 0.04 \mathrm{~m}^{2}\right.$ in cross-section area and $0.5 \mathrm{~m}$ in length); $C$ is the elastic joints which is simulated by two same circular cross-section short beams $(0.005 \mathrm{~m}$ in crosssection diameter and $0.05 \mathrm{~m}$ in length). Moreover, spatial beam element is used to mesh $P$ and $Q$, none-mass spatial beam element is used to mesh the elastic joints $C$. The element properties are listed in Tab. 1.

Table 1 Element properties of $P, Q$ and elastic joints $C$

\begin{tabular}{|c|c|c|c|c|}
\hline Structure & $\begin{array}{c}\text { Young's } \\
\text { modulus } \\
E / \mathrm{GPa}\end{array}$ & $\begin{array}{c}\text { Poisson's ratio } \\
\mu\end{array}$ & $\begin{array}{c}\text { Density } \\
\rho / \mathrm{kg} / \mathrm{m}^{3}\end{array}$ & $\begin{array}{c}\text { Element } \\
\text { length } \\
L / \mathrm{m}\end{array}$ \\
\hline$P$ and $Q$ & 70 & 0.3 & 2700 & 0.05 \\
\hline Elastic joints $C$ & 210 & 0.3 & 0 & 0.05 \\
\hline
\end{tabular}

Assuming that the damping of the example structure as illustrated in Fig. 3 is Rayleigh damping, the proportional coefficients can be defined as follows:

$\alpha=5 \times 10^{-3}, \beta=5 \times 10^{-6}$

By using the reformulated FBSM-CEJ to calculate the three translational direction FRFs between node $u$ and node $v$ as shown in Fig. 3, the example structure is divided into three substructures (namely substructure- $P$, substructure- $Q$ and substructure- $C$ ) and the former 40 order modes of both substructure- $P$ and substructure- $Q$ are reserved to calculate their respective FRF matrices. Via using the FEM to calculate the corresponding FRFs, all the modes of the example structure are taken into consideration. Additionally, for structure with Rayleigh damping, the calculation formula of its FRF matrix is given by [2]: 


$$
\boldsymbol{H}(\omega)=\sum_{i=1}^{w} \frac{\boldsymbol{\varphi}_{i} \boldsymbol{\varphi}_{i}^{\mathrm{T}}}{k_{i}+j \omega c_{i}-\omega^{2} m_{i}}
$$

where $\boldsymbol{\varphi}_{\boldsymbol{i}}, m_{i}, c_{i}$ and $k_{i}$ denote the $i$-th order mode and its corresponding modal mass, modal damping and modal stiffness of the structure, respectively. Meanwhile, the comparison results of the three translational direction FRFs between node $u$ and node $v$, obtained via the reformulated FBSM-CEJ and FEM respectively, are illustrated in Fig. 4.

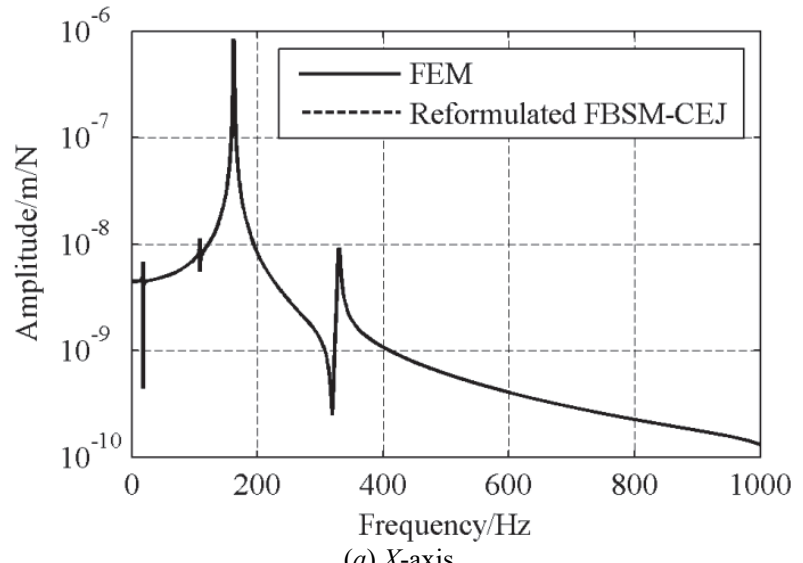

(a) $X$-axis

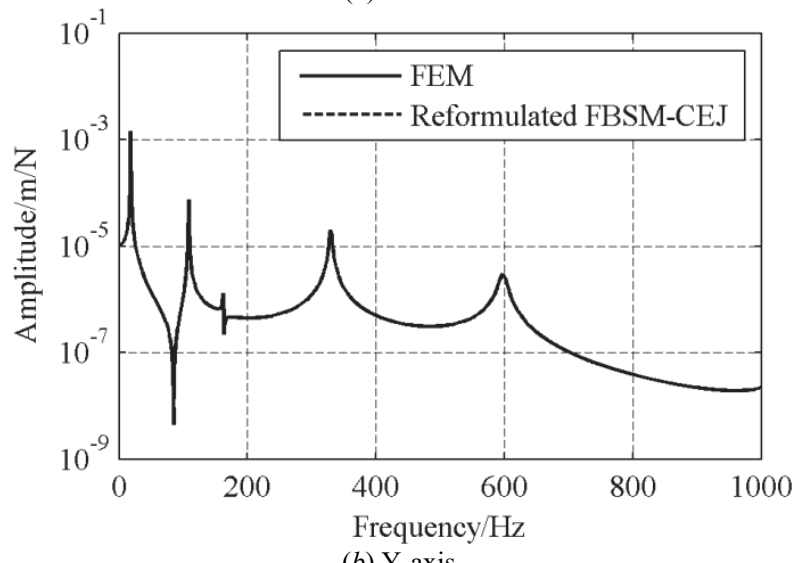

(b) $\mathrm{Y}$-axis

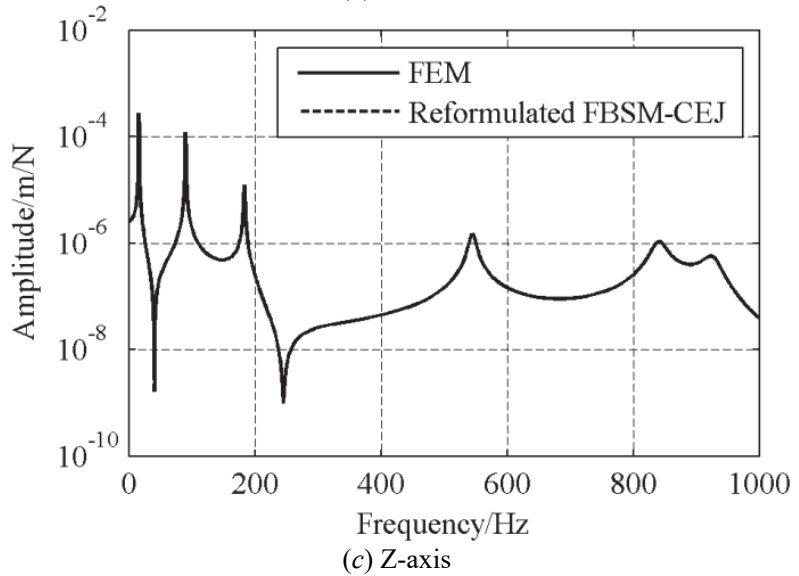

Figure 4 Comparison results of the three translational direction FRFs between node $u$ and node $v$

A significant conclusion can be drawn from Fig. 4 that the calculation results achieved via the reformulated FBSM-CEJ are in good agreement with those obtained by the FEM. Consequently, the reformulated FBSM-CEJ in this work has the advantages of both high precision and high efficiency.

\section{CONCLUSIONS}

According to all discussions above, the following conclusions can be formulated as:

(1) The FBSM-CEJ is reformulated according to SMWF in this paper. Comparing to the existing FBSMCEJ, the derivation process of the reformulated FBSM$\mathrm{CEJ}$ is more concise and the order of the matrix that requires inversion in the corresponding derivation result is lower. More important, the reformulated FBSM-CEJ possesses more applicability and operability, which can be used to directly and high-efficiently calculate the FRF matrix of a complex structure considering elastic joints with no matter singular or non-singular impedance matrix.

(2) Because of the existence of the stiffness coupling between the vertical translation and bending DOF in the stiffness matrix of the spatial beam element, the stiffness matrix of spatial beam element owns a higher accurate than 6-DOFs scalar spring system to simulate the stiffness of the elastic joints. In addition, as the mass of the elastic joints has almost no effect on the dynamic properties of the structure, using the none-mass spatial beam element to simulate the elastic joints is a more appropriate choice.

(3) Based on designing of an example structure with elastic joints, the performance of the reformulated FBSM$\mathrm{CEJ}$ is verified via numerical simulation. All the simulation results show that the reformulated FBSM-CEJ is able to calculate the FRF matrix of a structure considering elastic joints precisely.

In conclusion, all the achievements obtained from this article will provide not only high-efficiency, but also wideapplicability and facile-operability method for investigating the dynamic properties of the increasingly complex engineering structure considering elastic joints.

\section{REFERENCES}

[1] Chen, J. P. \&Cheng, W. (2016). Research on the disturbance generated by a solar array drive assembly driving a flexible system. Journal of Theoretical and Applied Mechanics, 54(3), 1001-1012. https://doi.org/10.15632/jtam-pl.54.3.1001

[2] Chen, J. P. (2017). Research on the micro-vibrations emitted by spacecraft moving equipment in uncertain boundary conditions. Dissertation for the Doctor and Philosophy Degree in Engineering of Beijing University of Aeronautics and Astronautics.

[3] Wang, M. \& Zheng, G. T. (2012). An improved fixedinterface modal synthesis method. Journal of astronautics, 33(3), 291-297.

[4] Wang, W. (2012). Research and implementation of frf-based substructure hybrid modelling. Dissertation for the Master Degree in Engineering of Huazhong University of Science and Technology.

[5] Klerk, D. D., Rixen, D. J., \& Voormeeren, S. N. (2008). General framework for dynamic substructuring: history, review, and classification of techniques. AIAA Journal, 46(5), 1169-1181. https://doi.org/10.2514/1.33274

[6] Wang, W., Hu Y. J. \& Ling, L. (2013). Method and implementation of substructure frf synthesis considering connection property. China Mechanical Engineering, 24(10), 1385-1389.

[7] Imregun, M., Robb, D. A., \& Ewins, D. J. (1987). Structural modification and coupling dynamic analysis using measured frf data. Proceedings of the Fifth International Modal Analysis Conference. 1136-1141. 
https://scholar.lanfanshu.cn/scholar?q=Structural+modification+an $d+$ coupling+dynamic+analysis+using+measured+frf+data

[8] Jetmundsen, B., Bielawa, R. L., \& Flannelly, W. G. (1987). Generalized frequency domain substructure synthesis. Journal of the American Helicopter Society, 33(1), 55-64. https://doi.org/info:doi/10.4050/JAHS.33.55

[9] Ren, Y. \& Beards, C. F. (1995). On substructure synthesis with frf data. Journal of Sound and Vibration, 185(5), 845866. https://doi.org/10.1006/jsvi.1995.0421

[10] Hu, Y. C. \& Chen, Z. W. (2008). Substructure synthesis method considering dynamic behavior of joints.Journal of Zhejiang University (engineering science), 42(8), 14041409.

[11] Ferreira, J. V. \& Ewins, D. J. (1996). Nonlinear receptance coupling approach based on describing functions. Proceedings of SPIE-The International Society for Optical Engineering. 1034-1040.

[12] Liu, W. (2001). Structural dynamic analysis and testing of coupled structures. Dissertation for the Doctor and Philosophy Degree in Engineering of Imperial College of Science, Technology and Medicine.

[13] Liu, W. \& Ewins D. J. (2002). Substructure synthesis via elastic media. Journal of Sound and Vibration, 257(2), 361379. https://doi.org/10.1006/jsvi.2002.5044

[14] Li, X. H. \& Yang, B. Y. (1987). The connecting substructures in the analysis of structural vibrations. Journal of astronautics, 8(1), 10-18.

[15] Zou, Y. J. (2010). Bush finite element principle and its application in building structural models for spacecraft. Spacecraft Engineering, 19(1), 99-105.

[16] Li, K. (2016). Research on spacecraft section vibration environment prediction methods. Dissertation for the Master Degree in Engineering of Nanjing University of Aeronautics and Astronautics.

[17] Golub, G. H. \& Van Loan, C. F. (1996). Matrix computations (third edition). Johns Hopkins University Press.

[18] Dohnal. F., Mace. B. R., \& Ferguson, N. S. (2009). Joint uncertainty propagation in linear structural dynamics using stochastic reduced basis methods. AIAA Journal, 47(4), 961969. https://doi.org/10.2514/1.38974

[19] Zhang, S. F. \& Zhao, D. (2012). Matrix theory tutorial (second edition). China Machine Press.

[20] Xing, Y. F. \& Li, M. (2011). Principles and methods of Computational Solid Mechanics. Beihang University Press.

[21] Chen, J. P., Wang, D., Liu, Y., Liu, Y., \& Zhang, W. W. (2018). Application of FRF based sub-structuring method in prediction of mechanical environment. Equipment Environmental Engineering, 15(11), 24-29.

\section{Contact information:}

\section{Jiangpan CHEN}

Beijing Institute of Electronic System Engineering, Beijing, No. 50 of Yongding Road, Haidian District, Beijing, China E-mail: chenjiangpan@hotmail.com

Yi LIU

Beijing Institute of Electronic System Engineering, Beijing No. 50 of Yongding Road, Haidian District, Beijing, China E-mail: Ishwifly@126.com

\section{Weiwen ZHANG}

Beijing Institute of Electronic System Engineering, Beijing, No. 50 of Yongding Road, Haidian District, Beijing, China E-mail: 504935834@126.com

Yan LIU

(Corresponding author)

Beijing Institute of Electronic System Engineering, Beijing, No. 50 of Yongding Road, Haidian District, Beijing, China E-mail: erica8027@163.com

\section{Dong WANG}

Beijing Institute of Electronic System Engineering, Beijing, No. 50 of Yongding Road, Haidian District, Beijing, China E-mail: 13520505058@163.com

\section{Limin SUN}

Beijing Institute of Electronic System Engineering, Beijing, No. 50 of Yongding Road, Haidian District, Beijing, China

E-mail: hebeisunlimin@163.com 